\title{
Noise in multivariate GPS position time-series
}

\author{
A. R. Amiri-Simkooei
}

Received: 26 February 2008 / Accepted: 15 July 2008 / Published online: 5 August 2008

(C) The Author(s) 2008

\begin{abstract}
A methodology is developed to analyze a multivariate linear model, which occurs in many geodetic and geophysical applications. Proper analysis of multivariate GPS coordinate time-series is considered to be an application. General, special, and more practical stochastic models are adopted to assess the noise characteristics of multivariate time-series. The least-squares variance component estimation (LS-VCE) is applied to estimate full covariance matrices among different series. For the special model, it is shown that the multivariate time-series can be estimated separately, and that the (cross) correlation between series propagates directly into the correlation between the corresponding parameters in the functional model. The time-series of five permanent GPS stations are used to show how the correlation between series propagates into the site velocities. The results subsequently conclude that the general model is close to the more practical model, for which an iterative algorithm is presented. The results also indicate that the correlation between series of different coordinate components per station is not significant. However, the spatial correlation between different stations for individual components is significant (a correlation of 0.9 over short baselines) both for white and for colored noise components.
\end{abstract}

Keywords Least-squares variance component estimation (LS-VCE) · Normal distribution · Multivariate GPS time-series $\cdot$ Spatial correlation

\section{A. R. Amiri-Simkooei $(\varangle)$}

Delft Institute of Earth Observation and Space Systems (DEOS), Faculty of Aerospace Engineering, Delft University of Technology, Kluyverweg 1, 2629 HS Delft, The Netherlands

e-mail: a.amirisimkooei@tudelft.nl

\section{A. R. Amiri-Simkooei}

Department of Surveying Engineering, Faculty of Engineering,

The University of Isfahan, 81744 Isfahan, Iran

\section{Introduction}

In geophysical studies, in addition to global models of plate motions, it is widely accepted that the site velocities of permanent GPS stations are determined by linear regression of individual GPS coordinate time-series. In an earlier work, a method is used to assess the noise characteristics of univariate GPS coordinate time-series (Amiri-Simkooei et al. 2007). A large number of permanent GPS stations allows one to apply a multivariate analysis method. This analysis includes both an optimal parameter estimation-site velocities for instanceand a realistic assessment of noise characteristics-variance and covariance components for instance-among different time-series. In multivariate models, the multiple dependent variables are measures of multiple outcomes, usually measured at the same point in time. A multivariate analysis might, for instance, be used to model the three coordinate components (north, east and up) at a single point in time.

If in a linear model, instead of one observation vector, there exist several observation vectors with identical covariance matrices, and the corresponding parameter vectors have to be determined, the model is referred to as a multivariate linear model. For the univariate linear model, in general, the covariance matrix of the observables is expressed as an unknown linear combination of some known cofactor matrices. One simple form (special case) of a covariance matrix is the presence of 1 unknown variance (of unit weight) in the stochastic model. The estimation of such unknowns is referred to as variance component estimation (VCE). This contribution generalizes the idea of VCE for a multivariate linear model.

We make use of the least-squares variance component estimation (LS-VCE) (Teunissen and Amiri-Simkooei 2008; Amiri-Simkooei 2007). When the observables are normally distributed, LS-VCE gives identical results with those of the 
many of the existing VCE methods such as best invariant quadratic unbiased estimator (BIQUE) (Koch 1978, 1999; Crocetto et al. 2000; Schaffrin 1981a, 1983; Caspary 1987), minimum norm quadratic unbiased estimator (MINQUE) (Rao 1971; Rao and Kleffe 1988; Sjöberg 1983; Xu et al. 2007), and restricted maximum likelihood (REML) estimator (Koch 1986). For applications of VCE-methods to GPS and geodetic data we refer to Chen et al. (1990), Kusche (2003a,b), Wang et al. (1998), Teunissen et al. (1998), Barnes (2002), Satirapod et al. (2002), Tiberius and Kenselaar (2000), Bona (2000), Bischoff et al. (2005, 2006), Fotopoulos (2005), $\mathrm{Xu}$ et al. (2006), Amiri-Simkooei (2007), Amiri-Simkooei and Tiberius (2007) and Schön and Brunner (2008a,b).

The VCE-methods have been applied to assess the noise characteristics of GPS position time-series. A realistic description of noise in GPS coordinate time-series is required to properly assess the error estimate of the unknown parameters. Several studies have recognized flicker noise (power-law noise with spectral index $\kappa=-1$ ) in addition to white noise in geodetic time-series (Zhang et al. 1997; Mao et al. 1999; Calais 1999; Williams et al. 2004; Nikolaidis et al. 2001; Teferle et al. 2008; Amiri-Simkooei et al. 2007). The last paper confirms, in addition, the presence of first-order autoregressive noise AR(1) in the series. Several researchers also acknowledge the presence of random walk noise $(\kappa=-2)$ or a combination of different noise components(Wyatt 1982; Johnson and Agnew 2000; Langbein and Johnson 1997; Langbein and Bock 2004; Langbein 2008). All these analysis are based on the univariate noise assessment for which the time-series are estimated individually.

In time series analysis of GPS coordinates, the noise components have been obtained by the maximum likelihood estimation (MLE), which is solved using the downhill simplex method (Press et al. 1992). In contrast to MLE, which gives biased estimators, LS-VCE provides unbiased and minimum variance estimators; MLE is only asymptotically unbiased, i.e., when the sample size is very large, which usually holds for GPS time series. Also, LS-VCE is much faster than MLE, as the downhill simplex method is extremely slow. With the LS-VCE method one is capable of applying hypothesis testing to the stochastic model. This allows one to judge, in an objective manner, which noise components are likely to be present in the data. The MLE method can provide the preferred noise model using the log-likelihood values, which are given after applying the method to the data.

Cross correlation between the time-series is considered to be an important issue. Williams et al. (2004) have reported significant spatial correlation between GPS time-series. In this contribution we elaborate this in detail. It is here worthwhile mentioning the related work on the developing and applying of the spatial filtering to the geophysical applications. We refer to Wdowinski et al. (1997), Nikolaidis (2002), Dong et al. (2006) and Teferle et al. (2006).
The objective of this paper is four-fold. First, we adopt three (general, special, and more practical) stochastic models to assess the noise characteristics of a multivariate linear model, with emphasis on GPS position time-series. Second, we provide an answer to the question as whether or not it is realistic to treat the GPS coordinate time-series separately (Amiri-Simkooei et al. 2007). Third, we elaborate the more practical formulation of the multivariate stochastic model that requires computation burden comparable to the univariate model. Fourth, the methodology is applied to the timeseries of five permanent GPS stations.

The article is organized as follows. Section 2 reviews the basic concepts of LS-VCE and univariate GPS coordinate time-series. Section 3 introduces a multivariate linear model along with its statistical analysis that occurs frequently in geodetic and geophysical applications. We start with the general formulation of the stochastic model, followed by a special case formulation, and finally by a more practical formulation, for which a straightforward and simple algorithm is provided. In Sect. 4, the noise characteristics of the multivariate GPS coordinate time-series are assessed. The emphasis in this section is on the spatial correlation of the series, both for white and for colored noise components.

\section{Univariate GPS coordinate time-series}

\subsection{Least-squares variance component estimation}

The LS-VCE is employed to assess the noise characteristics of GPS coordinate time-series. LS-VCE has many attractive features for which we refer to Teunissen and Amiri-Simkooei $(2008,2006)$ and Amiri-Simkooei (2007). Consider the following linear model of observation equations:

$\mathrm{E}(\underline{y})=A x, \quad \mathrm{D}(\underline{y})=Q_{y}=\sum_{k=1}^{p} \sigma_{k} Q_{k}$

where the $m \times n$ design matrix $A$ is assumed to be of full column rank, the $m \times m$ covariance matrix $Q_{y}$ of the $m$-observable vector $y$ is assumed to be positive definite, $x$, the $n$-vector of parameters has to be estimated and $\mathrm{E}$ and $\mathrm{D}$ are the expectation and dispersion operators, respectively (an underscore indicates a random variable).

The $m \times m$ cofactor matrices $Q_{k}$ are assumed to be symmetric such that the sum $\sum_{k=1}^{p} \sigma_{k} Q_{k}$ is positive definite. The cofactor matrices $Q_{k}, k=1, \ldots, p$ should be linearly independent, which is in fact the necessary condition for the stochastic model to have a regular solution. For more information we may refer to Amiri-Simkooei (2007) and Xu et al. (2007).

The least-squares estimator for the $p$-vector of unknown (co)variance components $\sigma=\left[\sigma_{1} \sigma_{2} \ldots \sigma_{p}\right]^{\mathrm{T}}$ can then be obtained as 
$\hat{\hat{\sigma}}=N^{-1} l$

with the $p \times p$ normal matrix $N$ and the $p$-vector $\underline{l}$ as

$n_{k l}=\frac{1}{2} \operatorname{tr}\left(Q_{y}^{-1} P_{A}^{\perp} Q_{k} Q_{y}^{-1} P_{A}^{\perp} Q_{l}\right)$

and

$\underline{l}_{k}=\frac{1}{2} \underline{\hat{e}}^{\mathrm{T}} Q_{y}^{-1} Q_{k} Q_{y}^{-1} \underline{\hat{e}}, \quad k, l=1,2, \ldots, p$

where $\underline{\hat{e}}=P_{A}^{\perp} y$ is the least-squares residuals and $P_{A}^{\perp}$ is an orthogonal projector given as

$P_{A}^{\perp}=I-A\left(A^{\mathrm{T}} Q_{y}^{-1} A\right)^{-1} A^{\mathrm{T}} Q_{y}^{-1}$

The (co)variance components can be obtained in an iterative procedure. We start with an initial guess for the (co)variance components. New updates are obtained in each iteration, and the procedure is repeated until the estimated components do not change with further iterations. Our conclusion regarding implementation of LS-VCE is that, at most, ten iterations are needed to obtain converged variance components.

Since the estimators $\underline{\hat{\sigma}}$ are based on the least-squares method, the inverse of the normal matrix $N$ automatically gives the covariance matrix of the estimated (co)variance components, namely $Q_{\hat{\sigma}}=N^{-1}$. This provides us with the measures of precision for the estimates.

The estimates obtained along with their precision should be presented as clearly as possible. For this purpose, visualizing techniques are appealing. Apart from that, when dealing with numbers, it is more convenient to demonstrate the numerical estimates in such a way that they are readily understandable. For example, if our original observations are expressed in unit of meter $(\mathrm{m})$, then the (co)variance components will be expressed in unit of $\mathrm{m}^{2}$ and the variance of these estimators in $\mathrm{m}^{4}$. It may not be convenient to deal with $\mathrm{m}^{2}$ and $\mathrm{m}^{4}$. In Appendix A we derive simple formulas for standard deviation estimators and correlation coefficients along with their precision only for the sake of presentation used, for instance, in Sect. 4

\subsection{Functional and stochastic models}

In this section, we consider individual GPS coordinate timeseries. One may use the daily solutions and estimate the parameters from time-series $i$. Consider a linear trend with $q-1$ periodic signals in the data series describing the deformation behavior and unmodelled periodic effects. The functional model $\mathrm{E}\left(\underline{y}_{i}\right)=A x_{i}$ then reads

$$
\begin{aligned}
\mathrm{E}\left(\underline{y}_{i}(t)\right)= & x_{i}^{(1)}+x_{i}^{(2)} t+\sum_{k=2}^{q} x_{i}^{(2 k-1)} \cos \omega_{k} t \\
& +x_{i}^{(2 k)} \sin \omega_{k} t
\end{aligned}
$$

where $\underline{y}_{i}$ is the $m$-vector of time-series observables, and the unknown $n$-vector $x_{i}$ consists of the intercept $x_{i}^{(1)}$, the slope $x_{i}^{(2)}$, and the coefficients $x_{i}^{(2 k-1)}$ and $x_{i}^{(2 k)}$ of the harmonic functions.

Examples of periodic patterns in the series are annual and semiannual signals, as well as signals with periods of 13.66, 14.2, and 14.8 days (Penna and Stewart 2003; Stewart et al. 2005; Penna et al. 2007). Recent studies (Amiri-Simkooei et al. 2007; Ray et al. 2007) on GPS coordinate time-series reveal the presence of other periodicities in the spectra (periods of 350 days and its fractions $350 / n, n=2, \ldots, 8)$. The design matrix $A$ is of size $m \times n$ where $n=2 q$. Another systematic error in GPS coordinates is the presence of jumps or offsets in the series; we refer to Williams (2003b), Kenyeres and Bruyninx (2004) and Perfetti (2006).

Without loss of generality, the covariance matrix of the time-series observations is chosen as

$\mathrm{D}\left(\underline{y}_{i}\right)=Q_{y_{i}}=\sigma_{i i}^{w} I+\sigma_{i i}^{f} Q_{f}$

where $I$ is an identity matrix of size $m$ - the cofactor matrix of white noise-and $Q_{f}$ is the cofactor matrix of flicker noise, for which the structure introduced by Zhang et al. (1997) is used. One can also use the Hosking flicker noise covariance matrix, which was introduced and used by Williams (2003a), Langbein (2004), Williams et al. (2004), Beavan (2005) and Bos et al. (2008). The flicker noise variances estimated in this paper are roughly one-half the size of those quoted in these papers (see Williams 2003a).

The least-squares estimator for $x_{i}$ is given as: $\hat{x}_{i}=\left(A^{\mathrm{T}}\right.$ $\left.Q_{y_{i}}^{-1} A\right)^{-1} A^{\mathrm{T}} Q_{y_{i}}^{-1} y_{i}$ with the covariance matrix of the form $Q_{\hat{x}_{i}}=\left(A^{\mathrm{T}} Q_{y_{i}}^{-1} A\right)^{-1}$. The LS-VCE method is employed to estimate the amplitudes of white noise (variance $\sigma_{i i}^{w}$ ) and flicker noise (variance $\sigma_{i i}^{f}$ ) in the time-series (see Eq. 2).

\section{Multivariate GPS coordinate time-series}

A significant and comparable amount of colored noise (between sites) reflects a common physical basis. Williams et al. (2004) showed plots of the (significant) spatial correlation as a function of angular distance. Reduction in both white and flicker noise from global solutions to regional solutions suggested that some of the noise is spatially correlated. The fact that different time-series can be correlated implies that it might not be realistic to estimate the series individually.

Most analysis of GPS time series estimate parameters from each series independent of other components. This has the advantage of being able to include all the colored (time-correlated) noise of the series and the disadvantage of neglecting the correlation between different time-series (e.g. spatial). One can neglect the time correlation and estimate only the between-series correlation. This has the disadvantage 
of giving too optimistic results for site velocity uncertainties and should be avoided (Zhang et al. 1997; Mao et al. 1999; Williams et al. 2004; Amiri-Simkooei et al. 2007).

The most sophisticated strategy, which gives more realistic results, is to include the time correlation as well as the between-series correlation. We can therefore estimate all parameters simultaneously using LS-VCE. We now consider three different possibilities for the stochastic model and explain the structure of each in detail.

\subsection{General model}

To keep the generality, we assume that the amounts of noise for white and flicker noise are different for different timeseries. Consider the following model consisting of $r$ time-series

$\mathrm{E}\left(\underline{y}_{i}\right)=A x_{i}, \mathrm{D}\left(\underline{y}_{i}, \underline{y}_{j}\right)=\sigma_{i j}^{w} I+\sigma_{i j}^{f} Q_{f}$

where $i$ and $j$ run from 1 to $r, y_{i}$ is the $m$-vector of observables for time-series $i$, and correspondingly $x_{i}$ is the $n$-vector of unknown parameters. The $m \times n$ design matrix $A$ and the $m \times m$ cofactor matrices $I$ and $Q_{f}$ are supposed to be identical for all time-series. $\mathbf{D}\left(\underline{y}_{i}, \underline{y}_{j}\right)$ is the (cross) covariance matrix between series $i$ and $j$.

The total number of observations and unknowns in the functional part of the model is $m r$ and $n r$, respectively. If one collects all unknown vectors $x_{i}$ in the $n \times r$ unknown matrix $X$, all observable vectors $y_{i}$ in the $m \times r$ observable matrix $\underline{Y}$, and correspondingly all residual vectors $\underline{e}_{i}$ in the $m \times r$ residual matrix $\underline{E}$, one obtains

$$
X=\left[x_{1} \ldots x_{r}\right] ; \quad \underline{Y}=\left[\underline{y}_{1} \ldots \underline{y}_{r}\right] ; \quad \underline{E}=\left[\underline{e}_{1} \ldots \underline{e}_{r}\right] .
$$

The unknowns in the stochastic model are the $2 \times r(r+$ $1) / 2=r(r+1)$ number of (co)variance elements of types $\sigma_{i j}^{w}$ and $\sigma_{i j}^{f}$. If one collects all (co)variance components $\sigma_{i j}^{w}$ and $\sigma_{i j}^{f}$ in the $r \times r$ matrices $\Sigma_{w}$ and $\Sigma_{f}$ respectively, then

$$
\Sigma_{w}=\left[\begin{array}{cccc}
\sigma_{11}^{w} & \sigma_{12}^{w} & \cdots & \sigma_{1 r}^{w} \\
\sigma_{12}^{w} & \sigma_{22}^{w} & \cdots & \sigma_{2 r}^{w} \\
\vdots & \vdots & \ddots & \vdots \\
\sigma_{1 r}^{w} & \sigma_{2 r}^{w} & \cdots & \sigma_{r r}^{w}
\end{array}\right] \quad \Sigma_{f}=\left[\begin{array}{cccc}
\sigma_{11}^{f} & \sigma_{12}^{f} & \cdots & \sigma_{1 r}^{f} \\
\sigma_{12}^{f} & \sigma_{22}^{f} & \cdots & \sigma_{2 r}^{f} \\
\vdots & \vdots & \ddots & \vdots \\
\sigma_{1 r}^{f} & \sigma_{2 r}^{f} & \cdots & \sigma_{r r}^{f}
\end{array}\right]
$$

With the preceding notations and using the properties of the vec-operator and the Kronecker product $\otimes$, one can rewrite Eq. (8) in a compact form as

$\mathrm{E}(\operatorname{vec}(\underline{Y}))=\left(I_{r} \otimes A\right) \operatorname{vec}(X)$

with the covariance matrix of the form

$\mathrm{D}(\operatorname{vec}(\underline{Y}))=Q_{\operatorname{vec}(Y)}=\Sigma_{w} \otimes I+\Sigma_{f} \otimes Q_{f}$ where $I_{r}$ is an identity matrix of size $r$. For the properties of the vec-operator and the Kronecker product $\otimes$ we refer to Magnus (1988); Amiri-Simkooei (2007). Equation (12) can in fact be generalized as $\mathrm{D}(\operatorname{vec}(\underline{Y}))=\sum_{k=1}^{p} \Sigma_{k} \otimes Q_{k}$. When $r=1$, this formulation reduces to the univariate model (see Eq. 7).

One can now apply the standard least-squares to estimate $X$ and LS-VCE to estimate the full unknown matrices $\Sigma_{w}$ and $\Sigma_{f}$. Since the number of observations $m$ of each series can be very large and the number of time series $r$ can be large, this method can be numerically expensive-one needs the successive inverses of the $m r \times m r$ matrix $Q_{\operatorname{vec}(Y)}$. However, when $r$ is small, say $r=2$ or $r=3$, numerical evaluation of the full formulation is still not very time-consuming.

One may consider $r=2$ to assess the noise characteristics of two time-series $\underline{y}_{1}$ and $\underline{y}_{2}$ simultaneously. One can then estimate the covariance matrix of the white noise and of the flicker noise components. There are in total six (co)variances for these two noise components to be estimated by LS-VCE, i.e. $r(r+1)=6$. In other words, the covariance matrix $\mathrm{D}=\mathrm{D}(\operatorname{vec}(\underline{Y}))$ of Eq. (12) reads

$$
\begin{aligned}
\mathrm{D}= & \sigma_{11}^{w}\left[\begin{array}{ll}
I & 0 \\
0 & 0
\end{array}\right]+\sigma_{22}^{w}\left[\begin{array}{ll}
0 & 0 \\
0 & I
\end{array}\right]+\sigma_{12}^{w}\left[\begin{array}{cc}
0 & I \\
I & 0
\end{array}\right] \\
& +\sigma_{11}^{f}\left[\begin{array}{cc}
Q_{f} & 0 \\
0 & 0
\end{array}\right]+\sigma_{22}^{f}\left[\begin{array}{cc}
0 & 0 \\
0 & Q_{f}
\end{array}\right]+\sigma_{12}^{f}\left[\begin{array}{cc}
0 & Q_{f} \\
Q_{f} & 0
\end{array}\right]
\end{aligned}
$$

LS-VCE can be applied - through Eqs. (2) to (4) - to estimate the (co)variances $\sigma_{11}^{w}, \sigma_{22}^{w}, \sigma_{12}^{w}, \sigma_{11}^{f}, \sigma_{22}^{f}$, and $\sigma_{12}^{f}$.

Given the preceding estimates, one can obtain the correlation coefficients (between series) of white noise and of flicker noise component as

$\hat{\rho}_{12}^{w}=\frac{\hat{\sigma}_{12}^{w}}{\sqrt{\hat{\sigma}_{11}^{w} \hat{\sigma}_{22}^{w}}}, \quad \hat{\rho}_{12}^{f}=\frac{\hat{\sigma}_{12}^{f}}{\sqrt{\hat{\sigma}_{11}^{f} \hat{\sigma}_{22}^{f}}}$

respectively. Because the covariance matrix of the (co) variance components is given by $N^{-1}$, one can simply obtain the variance of the correlation coefficients by applying the error propagation law to the linearized form of the preceding equations (as special case see later on Eq. 27).

\subsection{Special model}

We now consider a special structure $(p=1)$ of the stochastic model, which can simply be used for a large number of time-series (large $r$ ). The multivariate analysis of this special model turns out to be identical to the univariate analysis of the individual series. We also show how the significance of the correlation coefficients can be tested when the noise of the time-series is not necessarily white. 


\section{Parameter estimation}

Consider now a special case of Eq. (12) where there is only one noise component in the series, i.e., either white noise or flicker noise. It then follows that

$$
\mathrm{E}(\operatorname{vec}(\underline{Y}))=\left(I_{r} \otimes A\right) \operatorname{vec}(X), \quad \mathrm{D}(\operatorname{vec}(\underline{Y}))=\Sigma \otimes Q
$$

where the matrix $\Sigma$ plays the role of $\Sigma_{w}$ or $\Sigma_{f}$ and accordingly $Q$ plays the role of $I$ or $Q_{f}$.

To derive simplified formulas for the least-squares estimator of $X, Y$, and $E$, one needs simple expressions for the multivariate projectors $P_{I_{r} \otimes A}^{\perp}$ and $P_{I_{r} \otimes A}$ which follow as (see Amiri-Simkooei 2007, page 101)

$P_{I_{r} \otimes A}^{\perp}=I_{r} \otimes P_{A}^{\perp} ; \quad P_{I_{r} \otimes A}=I_{r} \otimes P_{A}$,

with univariate projectors $P_{A}=A\left(A^{\mathrm{T}} Q^{-1} A\right)^{-1} A^{\mathrm{T}} Q^{-1}$ and $P_{A}^{\perp}=I-P_{A}$. One can then show that the least-squares estimator of $X, Y$, and $E$ is

$\underline{\hat{X}}=\left(A^{\mathrm{T}} Q^{-1} A\right)^{-1} A^{\mathrm{T}} Q^{-1} \underline{Y} ; \quad \underline{\hat{Y}}=P_{A} \underline{Y} ; \quad \underline{\hat{E}}=P_{A}^{\perp} \underline{Y}$

respectively.

The preceding expressions are independent of the (un)known matrix $\Sigma$. This can be considered as a generalization of the univariate linear model $\mathrm{E}(\underline{y})=A x, \mathbf{D}(\underline{y})=$ $\sigma^{2} Q$, when the variance $\sigma^{2}$ of unit weight is (un)known. One can also show that the covariance matrix of the estimator $\operatorname{vec}(\underline{\hat{X}})$ is

$$
Q_{\operatorname{vec}(\hat{X})}=\Sigma \otimes\left(A^{\mathrm{T}} Q^{-1} A\right)^{-1}
$$

Equation (18) is also similar to the univariate model.

One can thus determine the outcomes of individual models separately. The unknown vector $x_{i}$ of the series $i$ and its (cross)covariance matrix are estimated as

$$
\hat{x}_{i}=\left(A^{\mathrm{T}} Q^{-1} A\right)^{-1} A^{\mathrm{T}} Q^{-1} y_{i}, \quad Q_{x_{i} x_{j}}=\sigma_{i j}\left(A^{\mathrm{T}} Q^{-1} A\right)^{-1}
$$

Let now $x_{i}^{(k)}$ be the $k$-th element of $x_{i}$ and $x_{j}^{(l)}$ the $l$-th element of $x_{j}$, then the variances of and the covariance between these elements read

$$
\sigma_{x_{i}^{(k)}}^{2}=\sigma_{i i} q_{k k} ; \quad \sigma_{x_{j}^{(l)}}^{2}=\sigma_{j j} q_{l l} ; \quad \sigma_{x_{i}^{(k)} x_{j}^{(l)}}=\sigma_{i j} q_{k l}
$$

where $q_{k l}$ denotes $\left(A^{\mathrm{T}} Q^{-1} A\right)^{-1}$ in index notation. The correlation coefficient $\rho_{i j}^{k l}$ between $x_{i}^{(k)}$ and $x_{j}^{(l)}$ then reads

$\rho_{i j}^{k l}=\frac{\sigma_{i j}}{\sqrt{\sigma_{i i} \sigma_{j j}}} \frac{q_{k l}}{\sqrt{q_{k k} q_{l l}}}=\frac{\sigma_{i j}}{\sigma_{i} \sigma_{j}} \frac{q_{k l}}{q_{k} q_{l}}=\rho_{i j} \rho^{k l}$

If one is interested in the correlation between elements of an individual series, i.e. if $i=j$, then $\rho_{i i}=1$. On the other hand, if one is interested in the correlation coefficient between an element in $x_{i}$ and its corresponding element in $x_{j}$, it will follow that $k=l$ and then $\rho^{k k}=1$. Therefore, one obtains

$\rho_{i j}^{k k}=\rho_{i j}=\frac{\sigma_{i j}}{\sigma_{i} \sigma_{j}}, \quad \rho_{i i}^{k l}=\rho^{k l}=\frac{q_{k l}}{q_{k} q_{l}}$

With the special model, the least-squares estimate of $x_{i}$ is obtained independent of other time-series. Also, the correlation between an element (e.g. site velocity) in $x_{i}$ and its corresponding element in $x_{j}$ is the same as the correlation between time-series $i$ and $j$, namely $\rho_{i j}$. Only for this special case does the correlation between time-series propagate directly into the correlation between parameters. This means that the time-series can be treated individually and the correlations between time-series can be added later into a covariance matrix of site velocities. We now have a theoretical proof-through Eqs. (20) to (22) —of the Williams et al. (2004) arguments.

\section{Variance-covariance estimation}

To obtain the covariance matrix of the estimators, so far the matrix $\Sigma$ was assumed to be known. If $\Sigma$ is unknown, one can rely on an estimate $\hat{\Sigma}$ instead. The minimum variance estimator of the unknown matrix $\Sigma$, obtained from LS-VCE, then reads (Amiri-Simkooei 2007; Teunissen and Amiri-Simkooei 2008; Schaffrin 1981b)

$\underline{\hat{\Sigma}}=\frac{\underline{\hat{E}}^{\mathrm{T}} Q^{-1} \underline{\hat{E}}}{m-n}$ with $\underline{\hat{E}}=P_{A}^{\perp} \underline{Y}=\left[\underline{\hat{e}}_{1} \underline{\hat{e}}_{2} \ldots \underline{\hat{e}}_{r}\right]$

where $m$-vectors $\underline{\hat{e}}_{i}, i=1,2, \ldots, r$ are the least-squares residual estimators of time-series $i$ obtained as $\underline{\hat{e}}_{i}=P_{A}^{\perp} \underline{y}_{i}$.

Because the method is based on the least-squares principle, one can also determine the precision description of the preceding (co)variance estimators. For time-series $i$ and $j$, the covariance matrix of the estimator vector $\left[\hat{\hat{\sigma}}_{i j} \underline{\hat{\sigma}}_{i i} \underline{\hat{\sigma}}_{j j}\right]^{\mathrm{T}}$ is given as (Amiri-Simkooei 2007)

$$
Q_{\hat{\sigma}}^{i j}=\frac{1}{m-n}\left[\begin{array}{crc}
\sigma_{i i} \sigma_{j j}+\sigma_{i j}^{2} & 2 \sigma_{i i} \sigma_{i j} & 2 \sigma_{j j} \sigma_{i j} \\
2 \sigma_{i i} \sigma_{i j} & 2 \sigma_{i i}^{2} & 2 \sigma_{i j}^{2} \\
2 \sigma_{j j} \sigma_{i j} & 2 \sigma_{i j}^{2} & 2 \sigma_{j j}^{2}
\end{array}\right]
$$

with $i, j=1,2, \ldots, r$. Note that all preceding estimators as well as their precision description are exact. Because the entries of $Q_{\hat{\sigma}}^{i j}$ are unknown a-priori, we have to be satisfied with an estimate $\hat{Q}_{\hat{\sigma}}^{i j}$ instead.

From $\hat{\Sigma}$, one can also compute the correlation coefficient between time-series (cf. Eq. 22)

$\hat{\rho}_{i j}=\frac{\hat{\sigma}_{i j}}{\sqrt{\hat{\sigma}_{i i} \hat{\sigma}_{j j}}}=\frac{\hat{\sigma}_{i j}}{\hat{\sigma}_{i} \hat{\sigma}_{j}}, \quad i, j=1,2, \ldots, r$

This is a nonlinear function of the variables $\hat{\sigma}_{i j}, \hat{\sigma}_{i i}$, and $\hat{\sigma}_{j j}$. Application of the error propagation law to the linearized form of the preceding equation yields $\sigma_{\hat{\rho}_{i j}}^{2}=J Q_{\hat{\sigma}}^{i j} J^{\mathrm{T}}$, where 
$J=\hat{\rho}_{i j}\left[\begin{array}{lll}\frac{1}{\hat{\sigma}_{i j}} & \frac{-1}{2 \hat{\sigma}_{i i}} & \frac{-1}{2 \hat{\sigma}_{j j}}\end{array}\right]$

is the Jacobian vector. The variance $\sigma_{\hat{\rho}_{i j}}^{2}$, with Eqs. (24) and (26), simplifies to

$\sigma_{\hat{\rho}_{i j}}^{2}=\frac{\left(1-\rho_{i j}^{2}\right)^{2}}{m-n}, \quad i, j=1,2, \ldots, r$

A formula for the correlation coefficient, for the case that two series $i$ and $j$ are uncorrelated and the number of common points $(m)$ is sufficiently large, is given by Press et al. (1992). The formula is expressed as $\sigma_{\hat{\rho}_{i j}}^{2}=1 / m$, which is a special case of Eq. (27) when $\rho_{i j}=0$ and $m \gg n$.

We now assume that $\underline{\hat{\rho}}_{i j}$ has a normal distribution, which for large $m$ (e.g. 500) is not unrealistic (Amiri-Simkooei 2007). We will then obtain

$\underline{\hat{\rho}}_{i j} \sim \mathrm{N}\left(\rho_{i j}, \sigma_{\hat{\rho}_{i j}}^{2}\right), \quad i, j=1,2, \ldots, r$

which can be used to test the significance of correlation coefficients (e.g. to test whether or not $\rho_{i j}=0$ ). The significance of correlations is traditionally tested with the implicit assumption that the two series are white.

Williams et al. (2004) simulated pairs of time-series with white and flicker noise to investigate the significance of spatial correlations. They concluded, for large $m$, that the standard deviation of the correlation coefficients tended to a steady value of less than 0.1 . This value guaranteed the significance of the estimated correlations. With the formulation described above it is now possible to test the significance of correlations with any type of noise as $Q$, introduced in Eq. (15), is an arbitrary positive definite matrix.

Example 1 (Identical structure of noise components) Assume that the structure of white and flicker noise is the same for different time-series. One then has

$\Sigma_{w}=\lambda_{w} \Sigma ; \Sigma_{f}=\lambda_{f} \Sigma$ with $\lambda_{w}$ and $\lambda_{f}$ known

meaning that the correlation matrix (between different timeseries) of the white noise component is the same as the flicker noise one. This can be the case when the correlation of the two noise components between time-series is the same (i.e. $\left.\rho_{i j}^{w}=\rho_{i j}^{f}, i, j=1, \ldots, r\right)$ and, in addition, the ratios of flicker noise to white noise amplitudes is the constant $\lambda_{f} / \lambda_{w}$.

The above structure for the covariance matrix can now be reduced to the formulation in Eq. (15) with

$Q=\lambda_{w} I+\lambda_{f} Q_{f}$

Other explanations and formulas go exactly along with those of the 'special model'. The above strategy is still a good approximation even when the above assumptions are mildly violated, for example, when the two series have slightly different ratios of flicker to white noise amplitudes or when the white noise correlation differs slightly from the flicker noise correlation; see also arguments of Williams et al. (2004).

\subsection{More practical model}

In Eq. (30) we assumed that $\lambda_{w}$ and $\lambda_{f}$ are known. In most practical applications, however, such parameters are unknown. To generalize Eq. (30), we now consider the stochastic model $\mathrm{D}(\operatorname{vec}(\underline{Y}))=\Sigma \otimes Q$ where $Q=\sum_{k=1}^{p} s_{k} Q_{k}$ is partly unknown, and both the matrix $\Sigma$ and the unknown factors $s_{k}$ are to be estimated using LS-VCE.

To solve the problem we first assume that $\Sigma$ is known. For the multivariate linear model we just need to substitute the terms in Eqs. (3) and (4) as follows: $\underline{\hat{e}} \leftarrow \operatorname{vec}(\underline{\hat{E}}), Q_{y} \leftarrow$ $\Sigma \otimes Q, Q_{k} \leftarrow \Sigma \otimes Q_{k}$, and $P_{A}^{\perp} \leftarrow I \otimes P_{A}^{\perp}$. After a few algebraic operations we obtain $\underline{\hat{s}}=N^{-1} \underline{l}$ where

$n_{k l}=\frac{r}{2} \operatorname{tr}\left(Q^{-1} P_{A}^{\perp} Q_{k} Q^{-1} P_{A}^{\perp} Q_{l}\right)$

and

$\underline{l}_{k}=\frac{1}{2} \operatorname{tr}\left(\underline{\hat{E}}^{\mathrm{T}} Q^{-1} Q_{k} Q^{-1} \underline{\hat{E}} \Sigma^{-1}\right)$

with the univariate projector $P_{A}^{\perp}=I-A\left(A^{\mathrm{T}} Q^{-1} A\right)^{-1} \times$ $A^{\mathrm{T}} Q^{-1}$.

Since $Q=\sum_{k=1}^{p} s_{k} Q_{k}$ is unknown a-priori, the unknown factors $s_{k}$ should be obtained through an iterative procedure. The advantage of this formulation over the general case formulation is that one needs the successive inverse of $Q$ which is of the size $m$ rather than $m r$. Therefore, if one includes more time-series in the model, the computational burden will not be increased much (it is similar to the univariate model).

In a special case where $\Sigma=I_{r}$, Eq. (32) simplifies to (cf. Eq. 4)

$\underline{l}_{k}=\frac{1}{2} \sum_{i=1}^{r} \underline{\underline{e}}_{i}^{\mathrm{T}} Q^{-1} Q_{k} Q^{-1} \underline{\hat{e}}_{i}=\sum_{i=1}^{r} \underline{\underline{l}}_{k}^{(i)}$

where superscripts ${ }^{(i)}$ refer to individual models. Equations (31) and (33) give

$\hat{s}=N^{-1} l=\frac{1}{r} \sum_{i=1}^{r} \hat{s}^{(i)}$

This equation shows, for $\Sigma=I_{r}$, that the unknown factors $s_{k}$ can be estimated as the arithmetic mean of the individual estimates. Such estimators have been introduced and used by Tiberius and Kenselaar (2003) and Schön and Brunner (2008b) to assess the noise characteristics of the GPS observables. This is considered as a theoretical proof for using such estimates.

If $\Sigma$ is unknown, the problem can be solved in a two-step procedure. For this purpose one first uses Eq. (23) to obtain an estimate for $\Sigma$, and then applies the preceding formulation. In other words, in Eq. (32) one can substitute $\hat{\Sigma}$ from Eq. (23) which yields

$\underline{l}_{k}=\frac{m-n}{2} \operatorname{tr}\left(\underline{\hat{E}}^{\mathrm{T}} Q^{-1} Q_{k} Q^{-1} \underline{\hat{E}}\left(\underline{\hat{E}}^{\mathrm{T}} Q^{-1} \underline{\hat{E}}\right)^{-1}\right)$ 


\section{Implementation of LS-VCE (multivariate model)}

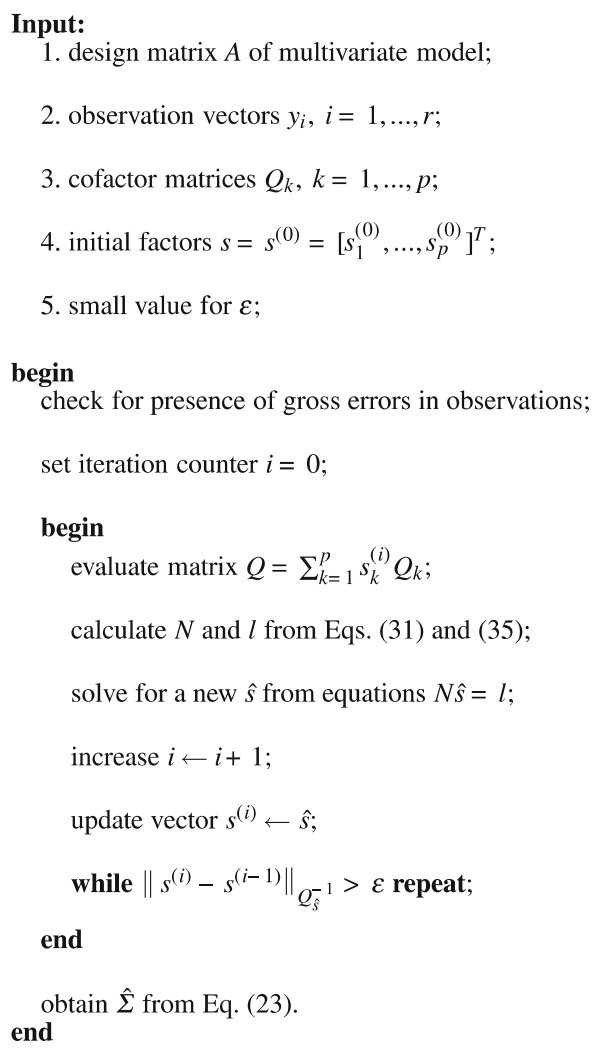

Fig. 1 Straightforward algorithm for implementation of least-squares variance component estimation in terms of a multivariate linear model $\mathrm{E}(\operatorname{vec}(\underline{Y}))=\left(I_{r} \otimes A\right) \operatorname{vec}(X)$ and $\mathrm{D}(\operatorname{vec}(\underline{Y}))=\Sigma \otimes \sum_{k=1}^{p} s_{k} Q_{k}$. The $s^{(i)}$ is the vector of unknown factors estimated in iteration $i$

Figure 1 gives a straightforward iterative algorithm for implementing a LS-VCE in terms of the multivariate model of observation equations.

\section{Applications, results and discussions}

The proposed multivariate analysis has been applied to two important applications of the daily GPS global solutions of permanent stations. The proper analysis of GPS time-series is an important issue in many geodetic and geophysical applications. The time-series of coordinates for KOSG, WSRT, ONSA, GRAZ and ALGO processed, using the precise point positioning (PPP) method in the GIPSY software (Zumberge et al. 1997), by the GPS Analysis Center at Jet Propulsion Laboratory (JPL) are adopted (Beutler et al. 1999). We have used 5 years of daily solutions for all sites (from 1999 to 2003).

We previously considered the univariate error analysis of these stations (Amiri-Simkooei et al. 2007). The results presented here are considered to be complementary to the results given in that paper. In both applications, the design matrix $A$ is obtained by the linear regression with annual and semiannual terms and signals with periods of $13.66,14.2$, and 14.8 days (Amiri-Simkooei et al. 2007).

\subsection{Correlation at one station}

One important issue related to the time-series is the (cross) correlation between coordinate components of a station. The components are suspected to be correlated since they are simultaneously estimated from the functional model based on the same set of (range) observations. This simultaneous estimation can lead to algebraic correlation among the estimators.

One of the applications of this method is to estimate the covariance matrix of one station consisting of three timeseries, namely, north, east, and up components. In this case, $r=3$, the design matrix $A$ has the same structure for the three time-series, and we use a simple stochastic model, namely $Q=I$ or $Q=Q_{f}$ in Eq. (15).

We estimated the covariance matrix and the correlation coefficients of the three coordinate components (Table 1) at individual sites using Eqs. (23) and (25), respectively. The correlations between different components do not seem to be significant. Insignificant correlations between components has also been shown by Bock et al. (1997).

We would have intuitively suspected that coordinate components of a station would be correlated. This correlation can be caused because the components are simultaneously estimated from the same data set through one functional model. The statement is however correct for one epoch of observations or for a couple of adjacent epochs. When considering all observations together $(24 \mathrm{~h})$, one has a well distributed satellite configuration with which the estimated coordinates will be approximately uncorrelated.

\subsection{Correlation between stations (spatial correlation)}

The formulations in Sect. 3 can also be applied to estimate the covariance matrix of an individual component (north, east or up) among different stations. One can thus determine how the solution for one particular station is correlated with those of other stations. We have estimated the spatial correlation, each time for one coordinate component and by three different stochastic models described in Sect. 3.

The first one is based on the supposition that the timeseries have only one noise component, e.g. either white or flicker noise (special model); the second one takes into account both white and flicker noise (general model) whereby we estimate one correlation coefficient for each noise component; and the third one uses the more practical formulation in which the matrix $\Sigma$ is fully unknown, and $Q$ is partly unknown. 
Table 1 Estimated standard deviation of north, east and up components as well as correlation coefficients between different components on assumption of $Q=I$; N: north, E: earth, and $\mathrm{U}$ : up component

\begin{tabular}{|c|c|c|c|c|c|c|}
\hline \multirow[b]{2}{*}{ Site code } & \multicolumn{3}{|c|}{ Standard deviation } & \multicolumn{3}{|c|}{ Correlation coefficient } \\
\hline & $\sigma_{N}(\mathrm{~mm})$ & $\sigma_{E}(\mathrm{~mm})$ & $\sigma_{U}(\mathrm{~mm})$ & $\rho_{N E}$ & $\rho_{N U}$ & $\rho_{E U}$ \\
\hline KOSG & $2.79 \pm 0.05$ & $3.07 \pm 0.06$ & $7.16 \pm 0.13$ & $-0.05 \pm 0.02$ & $-0.06 \pm 0.02$ & $-0.08 \pm 0.02$ \\
\hline WSRT & $2.76 \pm 0.05$ & $2.79 \pm 0.05$ & $7.16 \pm 0.13$ & $-0.06 \pm 0.02$ & $-0.06 \pm 0.02$ & $-0.02 \pm 0.02$ \\
\hline ONSA & $2.82 \pm 0.05$ & $2.90 \pm 0.05$ & $7.38 \pm 0.14$ & $0.10 \pm 0.02$ & $0.04 \pm 0.02$ & $0.01 \pm 0.02$ \\
\hline GRAZ & $3.02 \pm 0.06$ & $4.05 \pm 0.07$ & $8.33 \pm 0.15$ & $0.07 \pm 0.02$ & $-0.10 \pm 0.02$ & $-0.01 \pm 0.02$ \\
\hline ALGO & $2.93 \pm 0.05$ & $3.39 \pm 0.06$ & $7.19 \pm 0.13$ & $0.08 \pm 0.02$ & $-0.17 \pm 0.02$ & $0.03 \pm 0.02$ \\
\hline
\end{tabular}

Standard deviation of estimates is also included-special model

Table 2 Estimated spatial correlation coefficients (sorted by baseline length between stations) and their precision between corresponding north, east, and up component time-series for five stations $(Q=I)$

\begin{tabular}{llll}
\hline Distance $(\mathrm{km})$ & \multicolumn{3}{l}{ Correlation coefficient } \\
\cline { 2 - 4 } & North & \multicolumn{1}{l}{ East } & \multicolumn{1}{l}{ Up } \\
\hline 98 & $0.87 \pm 0.01$ & $0.69 \pm 0.01$ & $0.76 \pm 0.01$ \\
592 & $0.78 \pm 0.01$ & $0.60 \pm 0.02$ & $0.64 \pm 0.02$ \\
687 & $0.77 \pm 0.01$ & $0.56 \pm 0.02$ & $0.65 \pm 0.02$ \\
927 & $0.74 \pm 0.01$ & $0.43 \pm 0.02$ & $0.60 \pm 0.02$ \\
935 & $0.76 \pm 0.01$ & $0.45 \pm 0.02$ & $0.63 \pm 0.02$ \\
1,180 & $0.71 \pm 0.01$ & $0.38 \pm 0.02$ & $0.58 \pm 0.02$ \\
6,504 & $0.23 \pm 0.02$ & $-0.11 \pm 0.03$ & $-0.13 \pm 0.03$ \\
6,574 & $0.23 \pm 0.02$ & $-0.10 \pm 0.03$ & $-0.15 \pm 0.03$ \\
7,054 & $0.21 \pm 0.02$ & $-0.08 \pm 0.03$ & $-0.15 \pm 0.03$ \\
7,217 & $0.19 \pm 0.02$ & $-0.01 \pm 0.03$ & $-0.22 \pm 0.03$ \\
\hline
\end{tabular}

They also directly propagate into correlations between site velocitiesspecial model

\section{Special model}

For the special case, we may consider white or flicker noise in the series, i.e. $Q=I$ or $Q=Q_{f}$ in Eq. (15). There is no restriction for the number of the time-series used. One can simply estimate the variances and covariances between series of different sites using Eq. (23) where the least-squares residuals are $\hat{E}=P_{A}^{\perp} Y$ with $P_{A}^{\perp}=I-A\left(A^{\mathrm{T}} Q^{-1} A\right)^{-1} A^{\mathrm{T}} Q^{-1}$.

Table 2 gives the numerical results for $Q=I$, which presents only the spatial correlations between coordinate components. The results for $Q=Q_{f}$ are very similar to those for $Q=I$ and thus not repeated here. The correlation between time-series turns out to be significant. This is verified when one compares the correlations with their precision (use e.g. normal distribution in Eq. (28)). The significance of the correlations can also be simply a result from the Chebyschev inequality even when one does not specify a distribution.

The maximum correlations are obtained between the nearest sites, i.e. between KOSG and WSRT (they are only $98 \mathrm{~km}$
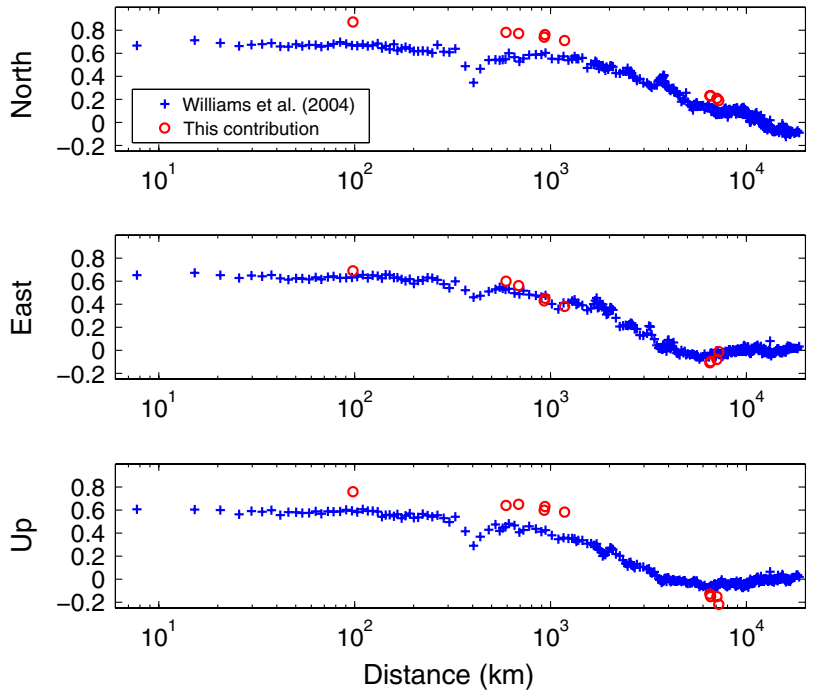

Fig. 2 Correlation coefficient of time series for north, east, and up components as a function of station separation. Pluses indicate the results from Williams et al. (2004) and circles indicate the results of this contribution

apart). This confirms that the noise has a common physical basis. Over the largest station separation (between ALGO and other sites), the spatial correlation is lower for the north component. It becomes negative for east and up components. It is important to note that the correlations given in Table 2 propagate directly into the correlation between the site velocities.

We now make a comparison (Fig. 2) with the results of the spatial correlations given by Williams et al. (2004), which was obtained from (S. D. P. Williams, Proudman Oceanographic Laboratory, personal communication, 2008). The correlations obtained here are slightly larger (in absolute sense) than those obtained by Williams. It is likely because we processed the newer part of the series which are less noisy than the older time-series precessed by Williams et al. (2004); the reduction of noise amplitude in the daily position estimates (toward the end of the series) was reported by Williams et al. (2004) and Amiri-Simkooei et al. (2007). 
Table 3 Estimated spatial correlation coefficients (sorted by baseline length) and their precision of white (top) noise and flicker (bottom) noise components between corresponding north, east, and up componentsgeneral model

\begin{tabular}{llrl}
\hline Distance $(\mathrm{km})$ & \multicolumn{2}{l}{ Correlation coefficient } \\
\cline { 2 - 4 } & \multicolumn{1}{l}{ North } & \multicolumn{1}{l}{ East } & \multicolumn{1}{l}{ Up } \\
\hline $98^{\mathrm{a}}$ & $0.84 \pm 0.01$ & $0.61 \pm 0.02$ & $0.68 \pm 0.02$ \\
592 & $0.79 \pm 0.02$ & $0.53 \pm 0.03$ & $0.53 \pm 0.04$ \\
687 & $0.78 \pm 0.02$ & $0.45 \pm 0.03$ & $0.50 \pm 0.04$ \\
927 & $0.74 \pm 0.02$ & $0.43 \pm 0.03$ & $0.45 \pm 0.04$ \\
935 & $0.78 \pm 0.02$ & $0.45 \pm 0.03$ & $0.47 \pm 0.04$ \\
1,180 & $0.72 \pm 0.02$ & $0.47 \pm 0.03$ & $0.34 \pm 0.05$ \\
6,504 & $0.26 \pm 0.04$ & $-0.17 \pm 0.04$ & $-0.06 \pm 0.05$ \\
6,574 & $0.30 \pm 0.04$ & $-0.13 \pm 0.04$ & $-0.08 \pm 0.05$ \\
7,054 & $0.25 \pm 0.04$ & $-0.15 \pm 0.04$ & $-0.06 \pm 0.05$ \\
7,217 & $0.33 \pm 0.04$ & $-0.08 \pm 0.04$ & $-0.07 \pm 0.05$ \\
$98^{\mathrm{b}}$ & $0.94 \pm 0.02$ & $0.90 \pm 0.03$ & $0.91 \pm 0.02$ \\
592 & $0.78 \pm 0.04$ & $0.81 \pm 0.05$ & $0.81 \pm 0.04$ \\
687 & $0.79 \pm 0.04$ & $0.77 \pm 0.06$ & $0.80 \pm 0.04$ \\
927 & $0.76 \pm 0.05$ & $0.49 \pm 0.09$ & $0.80 \pm 0.04$ \\
935 & $0.68 \pm 0.06$ & $0.44 \pm 0.10$ & $0.81 \pm 0.04$ \\
1,180 & $0.69 \pm 0.06$ & $0.34 \pm 0.10$ & $0.79 \pm 0.04$ \\
6,504 & $0.10 \pm 0.12$ & $-0.02 \pm 0.11$ & $-0.22 \pm 0.09$ \\
6,574 & $0.01 \pm 0.12$ & $-0.03 \pm 0.12$ & $-0.22 \pm 0.09$ \\
7,054 & $0.12 \pm 0.11$ & $0.01 \pm 0.11$ & $-0.27 \pm 0.09$ \\
7,217 & $-0.06 \pm 0.11$ & $-0.01 \pm 0.11$ & $-0.41 \pm 0.08$ \\
\hline & & & \\
\hline
\end{tabular}

${ }^{a}$ Spatial correlation of white noise

${ }^{\mathrm{b}}$ Spatial correlation of flicker noise

Such high correlations have significant effects on most geodetic and geophysical applications. For example, in geodetic applications-realization of ITRF for instance (see Altamimi et al. 2002) - they have significant effect on the estimation of the parameters of interest and their uncertainty. In geophysical applications they need to be taken into account for the proper interpretation and analysis of crustal deformation.

\section{General model}

The use of the general case formulation is restricted to the small values for $r$. We restrict ourselves to $r=2$ to assess the correlation of the white and of the flicker noise components (each time between two stations). There are in total six (co)variance components for two time-series of an individual component to be estimated by the general LS-VCE formulation (see Eq. 13). The final solution should be obtained through iteration.

Table 3 gives the spatial correlations of white (top) and flicker (bottom) noise components. The correlation coeffi-
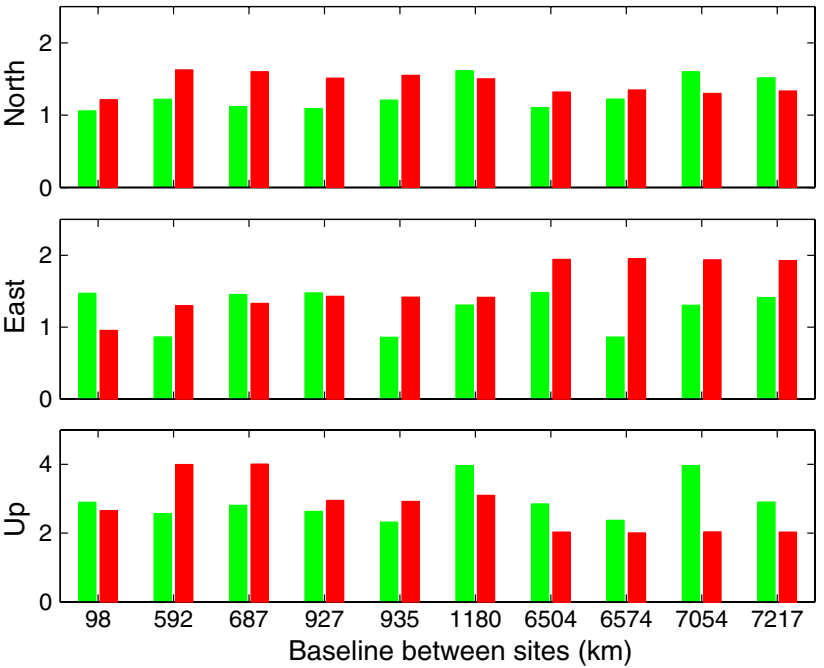

Fig. 3 Ratio of flicker noise to white noise amplitudes obtained from Eq. (13) for corresponding north, east, and up components of five permanent GPS stations; green (light): $\sigma_{11}^{f} / \sigma_{11}^{w}$ and red (dark): $\sigma_{22}^{f} / \sigma_{22}^{w}$; subscripts 1 and 2 indicate time-series 1 and 2 , respectively

cients in case of white noise (Table 2 for special model), seem, in general, to be between the two correlation values when we estimate one coefficient for each noise component. Both noise components seem to be spatially correlated to some extent. The spatial correlation of flicker noise (absolute values) is larger than that of white noise, on average, by a factor of 1.2. However, these differences do not seem to be significant when we compare them with the standard deviation of the correlation coefficients given in Tables 2 and 3.

Figure 3 shows the ratios of the flicker noise amplitudes to the white noise amplitudes for times series (between stations) of north, east, and up components. These ratios, on average, are approximately identical for mutual time-series (i.e. $\sigma_{11}^{f} / \sigma_{11}^{w} \equiv \sigma_{22}^{f} / \sigma_{22}^{w}$; the average of the red (dark) bars are approximately identical to the average of the green (light) bars). Also, parts of the variations are due to the negative correlation between the estimated flicker and white noise amplitudes. These all confirm that our formulation is close to the special case of Eq. (30) in Example 1.

Based on the general formulation of $Q_{\mathrm{vec}(Y)}$ in Eq. (12) and using the covariance matrix $Q_{\mathrm{vec}(X)}=\left(\left(I_{2} \otimes A^{\mathrm{T}}\right) Q_{\mathrm{vec}(Y)}^{-1}\right.$ $\left.\left(I_{2} \otimes A\right)\right)^{-1}$ of the parameters, we obtained the correlation coefficients between the site velocities (Table 4). They are very similar to those given in Table 3 for the flicker noise component. This makes sense since flicker noise is the dominating source of error in the series, and thus has the main contribution of the error on the parameters of interest as the site velocity. The values given in Table 4 have standard deviations comparable with those given in Table 3 for flicker noise component. This makes it easier to conclude that these results are not much different from those given in Table 2 for the site velocities. 
Table 4 Spatial correlation coefficients (sorted by baseline length) between site velocities for north, east, and up components - general model

\begin{tabular}{lccc}
\hline Distance $(\mathrm{km})$ & \multicolumn{3}{l}{ Correlation coefficient } \\
\cline { 2 - 4 } & North & East & Up \\
\hline 98 & 0.94 & 0.90 & 0.90 \\
592 & 0.78 & 0.80 & 0.81 \\
687 & 0.79 & 0.76 & 0.80 \\
927 & 0.76 & 0.49 & 0.79 \\
935 & 0.68 & 0.44 & 0.81 \\
1,180 & 0.69 & 0.34 & 0.79 \\
6,504 & 0.10 & -0.03 & -0.22 \\
6,574 & 0.02 & -0.03 & -0.22 \\
7,054 & 0.13 & -0.00 & -0.27 \\
7,217 & -0.06 & -0.01 & -0.40 \\
\hline
\end{tabular}

\section{More practical model}

The numerical evidence concluded that the structure of the covariance matrix is close to $\mathrm{D}(\operatorname{vec}(\underline{Y}))=\Sigma \otimes Q$ with $Q=s_{1} I+s_{2} Q_{f}$. For each coordinate component, $Y$ consists of the time-series observations of the five stations ( $\Sigma$ is of size $5 \times 5$ ). The iterative algorithm of Fig. 1 is now used to estimate both $\Sigma$ and $s_{1}$ and $s_{2}$. The correlation between series is given by $\Sigma$, and time correlation of the series is expressed by the relative magnitude of $s_{2}$ with respect to $s_{1}$.

The correlation coefficients obtained from $\Sigma$ are given in Table 5, and the amplitudes of white noise, i.e. multiplication of the diagonal elements of $\Sigma$ with $s_{1}$, are given in Table 6 . The amplitudes of flicker noise are correspondingly larger by factors of 1.14, 1.06, and 1.25 for north, east, and up components, respectively. The correlations are approximately identical to those given in Table 2 . This implies that the results are not dependent on the matrix $Q$, and therefore it is safe to use this stochastic model. Here also these correlations (between series) directly propagate into the correlations between site velocities.

\section{Concluding remarks}

In this contribution, for the multivariate linear model $\mathrm{E}(\operatorname{vec}(Y))=(I \otimes A) \operatorname{vec}(X)$, we considered the following stochastic models:

1. general model

$$
\mathrm{D}(\operatorname{vec}(\underline{Y}))=\sum_{k=1}^{p} \Sigma_{k} \otimes Q_{k}
$$

Table 5 Spatial correlations (sorted by baseline length between stations) and their precision between corresponding north, east, and up component time-series for five stations $\left(Q=s_{1} I+s_{2} Q_{f}\right)$

\begin{tabular}{llll}
\hline Distance $(\mathrm{km})$ & \multicolumn{2}{l}{ Correlation coefficient } \\
\cline { 2 - 4 } & North & East & \multicolumn{1}{l}{ Up } \\
\hline 98 & $0.86 \pm 0.01$ & $0.66 \pm 0.01$ & $0.76 \pm 0.01$ \\
592 & $0.78 \pm 0.01$ & $0.57 \pm 0.02$ & $0.63 \pm 0.02$ \\
687 & $0.78 \pm 0.01$ & $0.51 \pm 0.02$ & $0.61 \pm 0.02$ \\
927 & $0.74 \pm 0.01$ & $0.44 \pm 0.02$ & $0.57 \pm 0.02$ \\
935 & $0.75 \pm 0.01$ & $0.45 \pm 0.02$ & $0.59 \pm 0.02$ \\
1,180 & $0.71 \pm 0.01$ & $0.44 \pm 0.02$ & $0.52 \pm 0.02$ \\
6,504 & $0.23 \pm 0.02$ & $-0.14 \pm 0.03$ & $-0.11 \pm 0.03$ \\
6,574 & $0.24 \pm 0.02$ & $-0.12 \pm 0.03$ & $-0.12 \pm 0.03$ \\
7,054 & $0.22 \pm 0.02$ & $-0.11 \pm 0.03$ & $-0.13 \pm 0.03$ \\
7,217 & $0.24 \pm 0.02$ & $-0.06 \pm 0.03$ & $-0.17 \pm 0.03$ \\
\hline
\end{tabular}

The correlations also directly propagate into correlations between site velocities-more practical model

Table 6 Standard deviation estimates of white noise along with their precision for five stations $\left(Q=s_{1} I+s_{2} Q_{f}\right)$-more practical model

\begin{tabular}{|c|c|c|c|}
\hline \multirow[t]{2}{*}{ Site code } & \multicolumn{3}{|c|}{ Standard deviation } \\
\hline & $\sigma_{N}^{w} \quad(\mathrm{~mm})$ & $\sigma_{E}^{w} \quad(\mathrm{~mm})$ & $(\mathrm{mm})$ \\
\hline KOSG & $2.27 \pm 0.04$ & $2.52 \pm 0.05$ & $5.68 \pm 0.10$ \\
\hline WSRT & $2.22 \pm 0.04$ & $2.34 \pm 0.04$ & $5.59 \pm 0.10$ \\
\hline ONSA & $2.23 \pm 0.04$ & $2.36 \pm 0.04$ & $5.63 \pm 0.10$ \\
\hline GRAZ & $2.42 \pm 0.04$ & $3.12 \pm 0.06$ & $6.24 \pm 0.11$ \\
\hline ALGO & $2.38 \pm 0.04$ & $2.78 \pm 0.05$ & $5.81 \pm 0.11$ \\
\hline
\end{tabular}

2. $\quad$ special model $(p=1)$

$$
\mathrm{D}(\operatorname{vec}(\underline{Y}))=\Sigma \otimes Q
$$

3. more practical model $\left(\Sigma_{k}=s_{k} \Sigma\right)$

$$
\mathrm{D}(\operatorname{vec}(\underline{Y}))=\Sigma \otimes \sum_{k=1}^{p} s_{k} Q_{k}
$$

in which the matrices $\Sigma_{k}$ and the factors $s_{k}(k=1, \ldots, p)$ were estimated using LS-VCE.

We examined different GPS coordinates time-series together. In practice, it is more convenient to process time-series separately. There is a special model $(p=1)$ that gives identical results as to when we treat the time-series individually. The correlations between different time-series can simply be obtained from the least-squares residuals. The correlation between parameters - site velocities for instance-is then identical to the correlation between time-series observations.

The correlation between different components at one site is not significant. But, the correlation between different 
stations for individual components (spatial correlation) appeared to be significant over short distances (e.g., $100 \mathrm{~km}$ ). This holds both for white noise and flicker noise (general model). The coloured noise is the dominating source of error, and also, the correlations of the white noise are close to those of the flicker noise component. In addition the ratios of the flicker to white noise amplitudes are approximately identical for different time-series. These together confirm that the general formulation is close to the special model (Example 1).

Because the relative amplitudes of different noise components are usually unknown, it was recommended to employ the more practical model of the covariance matrix of the multivariate GPS coordinate time-series, i.e. $\mathrm{D}(\operatorname{vec}(\underline{Y}))=$ $\Sigma \otimes Q$ with $Q=\sum_{k=1}^{p} s_{k} Q_{k}$. Cross correlations-spatial correlation for instance-are given by the matrix $\Sigma$. Time correlation of the series are expressed by the components $s_{k}, k=1, \ldots, p$. The matrix $\Sigma$ as well as the components $s_{k}, k=1, \ldots, p$ can be estimated by LS-VCE using an iterative procedure (Fig. 1). The computational burden of this model is not much higher than the univariate model.

We noted that the final results are not seriously affected if we estimate the time-series separately. This conclusion suggested that the correlations between time-series can be added later into the covariance matrix of the parameters of interest. The amount of correlation is weakly dependent on the type of the stochastic model of the series. One may use a simple stochastic model — white noise only for instance- to obtain the correlation coefficients.

Acknowledgments I would like to acknowledge my colleagues Prof. P.J.G. Teunissen and Dr. C.C.J.M Tiberius for their useful discussions on an earlier version of this paper. I am also thankful to Prof. W. Featherstone, Dr. S.D.P. Williams and the anonymous reviewers for their helpful comments to significantly improve the presentation of the paper.

Open Access This article is distributed under the terms of the Creative Commons Attribution Noncommercial License which permits any noncommercial use, distribution, and reproduction in any medium, provided the original author(s) and source are credited.

\section{Appendix}

Presentation and interpretation of results

It is intended to derive simple formulas (by means of two lemmas) for standard deviation estimators and correlation coefficients along with their precision only for the sake of presentation of the (co)variance components.

Lemma 1 (Standard deviation estimator) Let $\hat{\sigma}_{i}^{2}=\hat{\sigma}_{i i}$ and $\sigma_{\hat{\sigma}_{i}^{2}}=\sigma_{\hat{\sigma}_{i i}}$ be the variance estimator and its standard deviation, respectively. They are both expressed in units of $\mathrm{m}^{2}$-as an example. To extract the more convenient indicators, we apply the square root to the variance estimator which gives the standard deviation estimator expressed in units of metres, namely

$\hat{\sigma}_{i}=\sqrt{\hat{\sigma}_{i}^{2}}=\sqrt{\hat{\sigma}_{i i}}$

It is possible to derive the precision of the variable $\hat{\sigma}_{i}$, namely $\sigma_{\hat{\sigma}_{i}}$, by applying the error propagation law to the nonlinear function. One can simply show that the precision of the standard deviation estimate, expressed in unit of $\mathrm{m}$, can be approximated using the following equation:

$\sigma_{\hat{\sigma}_{i}} \approx \frac{\sigma_{\hat{\sigma}_{i}^{2}}}{2 \hat{\sigma}_{i}}=\frac{\sigma_{\hat{\sigma}_{i i}}}{2 \hat{\sigma}_{i}}$

in which both $\hat{\sigma}_{i}$ and $\sigma_{\hat{\sigma}_{i i}}$ are given.

Lemma 2 (Correlation coefficient) Assume that we are given the covariance estimate $\hat{\sigma}_{i j}\left(\mathrm{~m}^{2}\right)$ and its precision $\sigma_{\hat{\sigma}_{i j}}\left(\mathrm{~m}^{2}\right)$ and two variance estimates $\hat{\sigma}_{i i}\left(\mathrm{~m}^{2}\right)$ and $\hat{\sigma}_{j j}\left(\mathrm{~m}^{2}\right)$ with their precision $\sigma_{\hat{\sigma}_{i i}}\left(\mathrm{~m}^{2}\right)$ and $\sigma_{\hat{\sigma}_{j j}}\left(m^{2}\right)$, respectively. In addition to the standard deviations of the estimates, there can also be covariances between estimates. The $3 \times 3$ matrix $Q_{\hat{\sigma}}^{i j}$ denotes the covariance matrix of the estimates.

In practice, it is more convenient to present the correlation coefficient rather than the covariance estimate, namely

$\hat{\rho}_{i j}=\frac{\hat{\sigma}_{i j}}{\hat{\sigma}_{i} \hat{\sigma}_{j}}=\frac{\hat{\sigma}_{i j}}{\sqrt{\hat{\sigma}_{i i}} \sqrt{\hat{\sigma}_{j j}}}$

To obtain the variance of the correlation coefficient $\hat{\rho}_{i j}$, we apply the error propagation law to the linearized form of the preceding equation. This then yields $\sigma_{\hat{\rho}}^{2}=J Q_{\hat{\sigma}}^{i j} J^{\mathrm{T}}$, where the Jacobian vector $J$ is given in Eq. (26).

\section{References}

Altamimi Z, Sillard P, Boucher C (2002) ITRF2000: a new release of the International Terrestrial Reference Frame for earth science applications. Journal of Geophysical Research 107(B10, 2214). doi:10.1029/2001JB000561

Amiri-Simkooei AR (2007) Least-squares variance component estimation: theory and GPS applications. PhD Thesis, Delft University of Technology, Publication on Geodesy, 64, Netherlands Geodetic Commission, Delft, http://repository.tudelft.nl/file/552363/ 372527

Amiri-Simkooei AR, Tiberius CCJM (2007) Assessing receiver noise using GPS short baseline time series. GPS Solut 11(1):21-35

Amiri-Simkooei AR, Tiberius CCJM, Teunissen PJG (2007) Assessment of noise in GPS coordinate time series: methodology and results. J Geophys Res 112:B07413. doi:10.1029/2006JB004913

Barnes JB (2002) Real time kinematic GPS and multipath: characterisation and improved least squares modelling. PhD Thesis, Department of Geomatics, University of Newcastle upon Tyne

Beavan J (2005) Noise properties of continuous GPS data from concrete pillar geodetic monuments in New Zealand and comparison with data from U.S. deep drilled braced monuments. J Geophys Res 110(B08410). doi:10.1029/2005JB003642 
Beutler G, Rothacher M, Schaer S, Springer TA, Kouba J, Neilan RE (1999) The International GPS Service (IGS): an interdisciplinary service in support of Earth sciences. Adv Space Res 23(4):631-635

Bischoff W, Heck B, Howind J, Teusch A (2005) A procedure for testing the assumption of homoscedasticity in least squares residuals: a case study of GPS carrier-phase observations. J Geod 78: 397-404

Bischoff W, Heck B, Howind J, Teusch A (2006) A procedure for estimating the variance function of linear models and for checking the appropriateness of estimated variances: a case study of GPS carrier-phase observations. J Geod 79:694-704

Bock Y, Wdowinski S, Fang P, Zhang J, Williams S, Johnson H, Behr J, Genrich J, Dean J, Domselaar M , van Agnew D, Wyatt F, Stark K, Oral B, Hudnut K, King R, Herring T, Dinardo S, Young W, Jackson D, Gurtner W (1997) Southern California Permanent GPS Geodetic Array: Continuous measurements of regional crustal deformation between the 1992 Landers and 1994 Northridge earthquakes. J Geophys Res 102(B8):18013-18033

Bona P (2000) Precision, cross correlation, and time correlation of GPS phase and code observations. GPS Solut 4(2):3-13

Bos MS, Fernandes RMS, Williams SDP, Bastos L (2008) Fast error analysis of continuous GPS observations. J Geod 82:157-166. doi:10.1007/s00190-007-0165-x

Calais E (1999) Continuous GPS measurements across the Western Alps, 1996-1998. Geophys J Int 138:221-230

Caspary WF (1987) Concepts of network and deformation analysis. Tech. rep., School of Surveying, The University of New South Wales, Kensington

Chen YQ, Chrzanowski A, Kavouras M (1990) Assessment of observations using minimum norm quadratic unbiased estimation (MINQUE). CISM J ACSGS 44:39-46

Crocetto N, Gatti M, Russo P (2000) Simplified formulae for the BIQUE estimation of variance components in disjunctive observation groups. J Geod 74:447-457

Dong D, Fang P, Bock Y, Webb F, Prawirodirdjo L, Kedar S, Jamason P (2006) Spatiotemporal filtering using principal component analysis and Karhunen-Loeve expansion approaches for regional GPS network analysis. J Geophys Res 111:B03405. doi:10.01029/ 02005JB003806

Fotopoulos G (2005) Calibration of geoid error models via a combined adjustment of ellipsoidal, orthometric and gravimetric geoid height data. J Geod 79:111-123, doi:10.1007/s00190-005-0449-y

Johnson HO, Agnew DC (2000) Correlated noise in geodetic time series. U.S. Geol. Surv. Final Tech. Rep., FTR-1434-HQ-97-GR-03155

Kenyeres A, Bruyninx C (2004) EPN coordinate time series monitoring for reference frame maintenance. GPS Solut 8:200-209. doi:10. 1007/s10291-004-0104-8

Koch KR (1978) Schätzung von Varianzkomponenten. Allgemeine Vermessungs Nachrichten 85:264-269

Koch KR (1986) Maximum likelihood estimate of variance components. Bull Géod 60:329-338, ideas by A.J. Pope

Koch KR (1999) Parameter estimation and hypothesis testing in linear models. Springer, Berlin

Kusche J (2003a) A Monte-Carlo technique for weight estimation in satellite geodesy. J Geod 76:641-652

Kusche J (2003b) Noise variance estimation and optimal weight determination for GOCE gravity recovery. Adv Geosci 1:81-85

Langbein J (2004) Noise in two-color electronic distance meter measurements revisited. J Geophys Res 109:B04406. doi:10.1029/ 2003JB002819

Langbein J (2008) Noise in GPS displacement measurements from southern california and southern nevada. J Geophys Res. doi:10.1029/ 2007JB005247 (in press)

Langbein J, Bock Y (2004) High-rate real-time GPS network at Parkfield: Utility for detecting fault slip and seismic displacements. Geophys Res Lett 31:L15S20. doi:10.1029/2003GL019408
Langbein J, Johnson H (1997) Correlated errors in geodetic time series: implications for time-dependent deformation. J Geophys Res 102(B1):591-603

Magnus JR (1988) Linear structures. Oxford University Press, London School of Economics and Political Science, Charles Griffin \& Company LTD, London

Mao A, Harrison CGA, Dixon TH (1999) Noise in GPS coordinate time series. J Geophys Res 104(B2):2797-2816

Nikolaidis R, Bock Y, de Jonge PJ, Shearer P, Agnew DC, Domselaar M (2001) Seismic wave observations with the global positioning system. J Geophys Res 106:21,897-21,916

Nikolaidis RM (2002) Observation of geodetic and seismic deformation with the global positioning system. PhD Thesis, University of California, San Diego

Penna NT, Stewart MP (2003) Aliased tidal signatures in continuous GPS height time series. Geophys Res Lett 30(23):2184. doi:10. 1029/2003GL018828

Penna NT, King MA, Stewart MP (2007) GPS height time series: Shortperiod origins of spurious long-period signals. J Geophys Res 112(B02402). doi:10.1029/2005JB004047

Perfetti N (2006) Detection of station coordinate discontinuities within the Italian GPS Fiducial Network. J Geod 80(7):381-396. doi:10. 1007/s00190-006-0080-6

van Press WH, Flannery BP, Teukolsky SA, Vetterling WT (1992) Numerical Recipes. Cambridge University Press, New York

Rao CR (1971) Estimation of variance and covariance componentsMINQUE theory. J Multivariate Anal 1:257-275

Rao CR, Kleffe J (1988) Estimation of variance components and applications, vol 3. Series in Statistics and Probability, North-Holland

Ray J, Altamimi Z, Collilieux X, van Dam T (2007) Anomalous harmonics in the spectra of GPS position estimates. GPS Solut doi:10. 1007/s10291-007-0067-7

Satirapod C, Wang J, Rizos C (2002) A simplified MINQUE procedure for the estimation of variance-covariance components of GPS observables. Surv Rev 36(286):582-590

Schaffrin B (1981a) Ausgleichung mit Bedingungs-Ungleichungen. Allgemeine Vermessungs Nachrichten 88:227-238

Schaffrin B (1981b) Best invariant covariance component estimators and its application to the generalized multivariate adjustment of heterogeneous deformation observations. Bull Géod 55:73-85

Schaffrin B (1983) Varianz-kovarianz-komponenten-schätzung bei der ausgleichung heterogener wiederholungsmessungen C282, Deutsche Geodätische Kommission, München

Schön S, Brunner FK (2008a) Atmospheric turbulence theory applied to GPS carrier-phase data. J Geod 82(1):47-57

Schön S, Brunner FK (2008b) A proposal for modelling physical correlations of GPS phase observations. J Geod. doi:10.1007/ s00190-008-0211-3 (in press)

Sjöberg LE (1983) Unbiased estimation of variance-covariance components in condition adjustment with unknowns - a MINQUE approach. Zeits ür Vermessungswesen 108(9):382-387

Stewart MP, Penna NT, Lichti DD (2005) Investigating the propagation mechanism of unmodelled systematic errors on coordinate time series estimated using least squares. J Geod 79:479-489. doi:10. 1007/s00190-005-0478-6

Teferle FN, Bingley R, Williams SDP, Baker T, Dodson A (2006) Using continuous GPS and absolute gravity to separate vertical land movements and changes in sea level at tide gauges in the UK. Philos Trans Roy Soc A 364:917-930

Teferle FN, Williams SDP, Kierulf HP, Bingley RM, Plag HP (2008) A continuous GPS coordinate time series analysis strategy for highaccuracy vertical land movements. Phys Chem Earth 33:205216

Teunissen PJG, Amiri-Simkooei AR (2006) Variance component estimation by the method of least-squares. In: Xu P, Liu J, Dermanis A (eds) VI Hotine-Marussi symposium of theoretical and compu- 
tational geodesy, IAG Symposia, vol 132, 29 May-2 June, 2006, China. 132. Springer, Berlin, 273-279

Teunissen PJG, Amiri-Simkooei AR (2008) Least-squares variance component estimation. J Geod 82(2):65-82. doi:10.1007/ s00190-007-0157-x

Teunissen PJG, Jonkman NF, Tiberius CCJM (1998) Weighting GPS dual frequency observations: bearing the cross of cross-correlation. GPS Solut 2(2):28-37

Tiberius CCJM, Kenselaar F (2000) Estimation of the stochastic model for GPS code and phase observables. Surv Rev 35(277):441-454

Tiberius CCJM, Kenselaar F (2003) Variance component estimation and precise GPS positioning: case study. J Surv Eng 129(1):11-18

Wang J, Stewart MP, Tsakiri M (1998) Stochastic modeling for static GPS baseline data processing. J Surv Eng 124(4):171-181

Wdowinski S, Bock Y, Zhang J, Fang P, Genrich J (1997) Southern california permanent GPS geodetic array: spatial filtering of daily positions for estimating coseismic and postseismic displacements induced by the 1992 landers earthquake. J Geophys Res 102:18,05718,070

Williams SDP (2003a) The effect of coloured noise on the uncertainties of rates estimated from geodetic time series. J Geod 76:483-494
Williams SDP (2003b) Offsets in global positioning system time series. J Geophys Res 108(B6):2310. doi:10.1029/2002JB002156

Williams SDP, Bock Y, Fang P, Jamason P, Nikolaidis RM, Prawirodirdjo L, Miller M, Johnson DJ (2004) Error analysis of continuous GPS position time series. J Geophys Res 109:B03412. doi:10. 1029/2003JB002741

Wyatt FK (1982) Displacement of surface monuments: horizontal motion. J Geophys Res 87:979-989

Xu PL, Shen YZ, Fukuda Y, Liu YM (2006) Variance component estimation in linear inverse ill-posed models. J Geod 80:69-81

Xu PL, Liu YM, Shen YZ (2007) Estimability analysis of variance and covariance components. J Geod 81:593-602. doi:10.1007/ s00190-006-0122-0

Zhang J, Bock Y, Johnson H, Fang P, Williams S, Genrich J, Wdowinski S, Behr J (1997) Southern California permanent GPS geodetic array: error analysis of daily position estimates and site velocitties. J Geophys Res 102:18035-18055

Zumberge JF, Heflin MB, Jefferson DC, Watkins MM, Webb FH (1997) Precise point positioning for the efficient and robust analysis of GPS data from large networks. J Geophys Res 102:5005-5017 